\title{
LA EVOLUCIÓN DE LAS PRECIPITACIONES EN LA CUENCA OCCIDENTAL DEL MEDITERRÁNEO: ¿TENDENCIA O CICLOS? ${ }^{1}$
}

\author{
J. Quereda Sala, E. Montón Chiva y J. Escrig Barberá \\ Estación de Climatología Aplicada (Univ. Jaume I, Castellón)
}

\section{RESUMEN}

El presente trabajo trata de responder a dos de las más inquietantes cuestiones que actualmente se plantean a la Climatología mediterránea. Estas interrogantes son: ¿Acaso están disminuyendo las precipitaciones de la región mediterránea? y, asimismo, ¿Está esa evolución pluviométrica sujeta a ciclos? La respuesta a ambas cuestiones se fundamenta en el análisis de las series de los 37 principales observatorios históricos, con datos anteriores al siglo XX, enclavados en el entorno de la Cuenca Occidental del Mediterráneo.

Palabras clave: cambio climático, precipitación, media anual, tendencias, Cuenca Occidental del Mediterráneo.

\section{SUMMARY}

The main goal of this paper tries to answer two of the more worring questions at the moment about Mediterranean Climatology. These questions are: Is the raifall of the Mediterranean region decreasing?, and anyway, Is that precipitation evolution subject to cycles? The answer to both questions is based on the analysis of the series from the 37 main historic observatories which began before the 20th century in the Western Mediterranean Basin. Basin.

Key words: climatic change, rainfall, annual average, trends, Western Mediterranean

\section{RÉSUMÉ}

L'objectif du présent étude n'est pas outre qu'essayer de répondre à deux des plus inquiétantes questions que la Climatologie méditerranénne à actuellement posées. Ces deux questions sont les suivantes: Y a-t-il vraiment une tendance à une diminution des

1 Este trabajo corresponde a la ponencia presentada en las Jornadas Científicas sobre «Sequías en España» organizadas por el Instituto Universitario de Geografía de la Universidad de Alicante en noviembre de 1999. 
pluies? Et, est-il posssible que cette evolution soit cyclique? Cette analyse est portée sur les series temporelles des 37 principaux observatoires avec des enregistrements antérieurs au XX ème siècle, à la Méditerranée Occidentale.

Mots clés: change climatique, cumuls pluviométriques annuels, tendances, Méditerranée Occidentale.

\section{La importancia de la cuestión: ¿Están disminuyendo las precipitaciones de la región mediterránea?}

Con toda sinceridad habremos de convenir que lo que realmente se busca tras un título como el que encabeza estas líneas «La evolución de las precipitaciones en la cuenca occidental del Mediterráneo» es la respuesta a una de las más inquietantes cuestiones o retos científicos que tiene ante sí la Climatología mediterránea. Esta es propiamente la cuestión: ¿Están disminuyendo las precipitaciones de la región mediterránea?

Una interrogante que tanto administración como gran público plantean insistentemente a los profesionales de las ciencias de la atmósfera, ante la evolución pluviométrica de los últimos años. Los diversos períodos de sequía registrados, especialmente los 1978-1982, 1992-1995 y el actual, parecen magnificarse e incluso anular la memoria de que esa preocupación por los recursos hídricos no es nada nuevo sino que ha sido ancestral en nuestras tierras. En ellas, la mayor parte de las horas de vigilia de su prominente y extenso sector agrícola han estado ocupadas por las exigencias en agua de unos cultivos que, desde siempre, han sido base de su economía. Exigencias acrecentadas con el desarrollo demográfico y económico modernos y que han planteado un auténtico reto investigador en los momentos en que las hipótesis sobre un cambio climático reducen las previsiones pluviométricas en valores del 20 al $25 \%$ para los albores del siglo XXI.

De este modo, hoy, a finales de 1999, nos hallamos ante una situación de sequía semejante a las anteriores o tal vez incluso más angustiosa. ¿Cómo hemos llegado a ella? ¿Acaso es real esa tendencia hacia la disminución de lluvias como corolario de un cambio climático?

\section{La hipótesis del cambio climático}

Las mayores alteraciones ambientales previstas para los próximos cincuenta o cien años vendrían asociadas al cambio climático producido por el incremento en la concentración atmosférica de dióxido de carbono y otros gases. Este cambio climático produciría notables transtornos biogeográficos y del orden económico-social. A este respecto, los últimos modelos preconizan que las temperaturas globales podrían incrementarse entre 1,5 y $4,5^{\circ} \mathrm{C}$. Un incremento térmico que, por otra parte, no sería la única respuesta atmosférica al nuevo ajuste del balance de radiación sobre el planeta. Simultáneamente, el sistema climático se vería afectado por sensibles alteraciones en el régimen pluviométrico.

La Cuenca Occidental del Mediterráneo, en el borde meridional de la zona templada entre $\operatorname{los} 35^{\circ} \mathrm{N}$ y $\operatorname{los} 45^{\circ} \mathrm{N}$, podría estar abocada, de este modo, a experimentar impactos climáticos y biogeográficos de gran intensidad. En consecuencia, a lo largo del siglo XXI, se podría asistir a una sensible readaptación de su escenario biogeográfico muy afectado por el aumento de las evapotranspiraciones y disminución de los recursos pluviométricos. Procesos ambos que los actuales modelos preconizan en base al progresivo alejamiento de la zona frontogenética polar (Fig. 1). 


\section{PRECIPTIATION CHANGE (mmiday) GHG1-Contiol 203X-195X Ann}

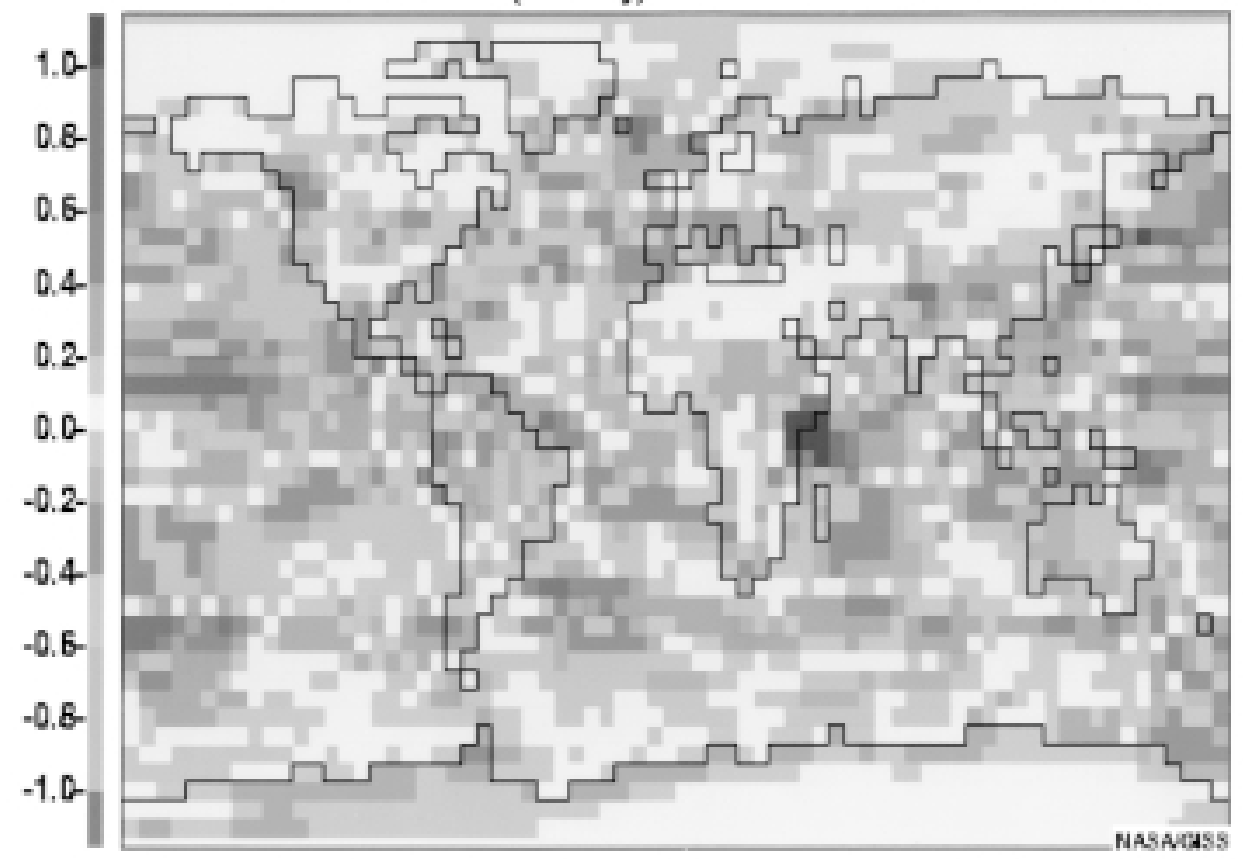

FiguRA 1. Escenario climático previsto por el IPCC'1998 para el año 2025: Simulación a 30 años del Atmosphere-Ocean Model con un incremento del $\mathrm{CO}_{2}$ del $1 \%$ anual, usado por el United Nations Intergovernmental Panel on Climate Change (IPCC) para su Second Scientific Assessment of Climate. La región mediterránea podría asistir a una sensible reducción de sus precipitaciones.

\section{¿Se puede comprobar ya esta hipótesis?, ¿Se está registrando ya una reducción de los recursos pluviométricos?}

Estas son las grandes interrogantes que la Climatología tiene ante sí para dar respuesta a una demanda social, especialmente angustiada en épocas de sequía como la actual. Y la verdad sea dicha, parece que no debería tener dificultades en pronunciarse ya que cuenta con numerosos observatorios que vienen registrando las precipitaciones desde mediados del siglo pasado.

Sin embargo, la realidad es muy distinta. En efecto, si bien disponemos de observatorios suficientes en cantidad para comprobar el comportamiento y evolución de las precipitaciones, no sucede lo mismo respecto a la calidad de los datos que contienen. Es decir, que si bien todas estas series pluviométricas son aceptables en cuanto a duración y continuidad, no lo son tanto respecto a la posible calidad de sus datos.

\section{Los observatorios históricos y sus series pluviométricas: métodos de análisis}

El análisis de la evolución pluviométrica mediterránea que aquí se realiza está basado en las series de aquellos observatorios históricos que iniciaron sus registros con anterioridad al siglo XX. Estos observatorios históricos, 37 en total, aparecen localizados en la 


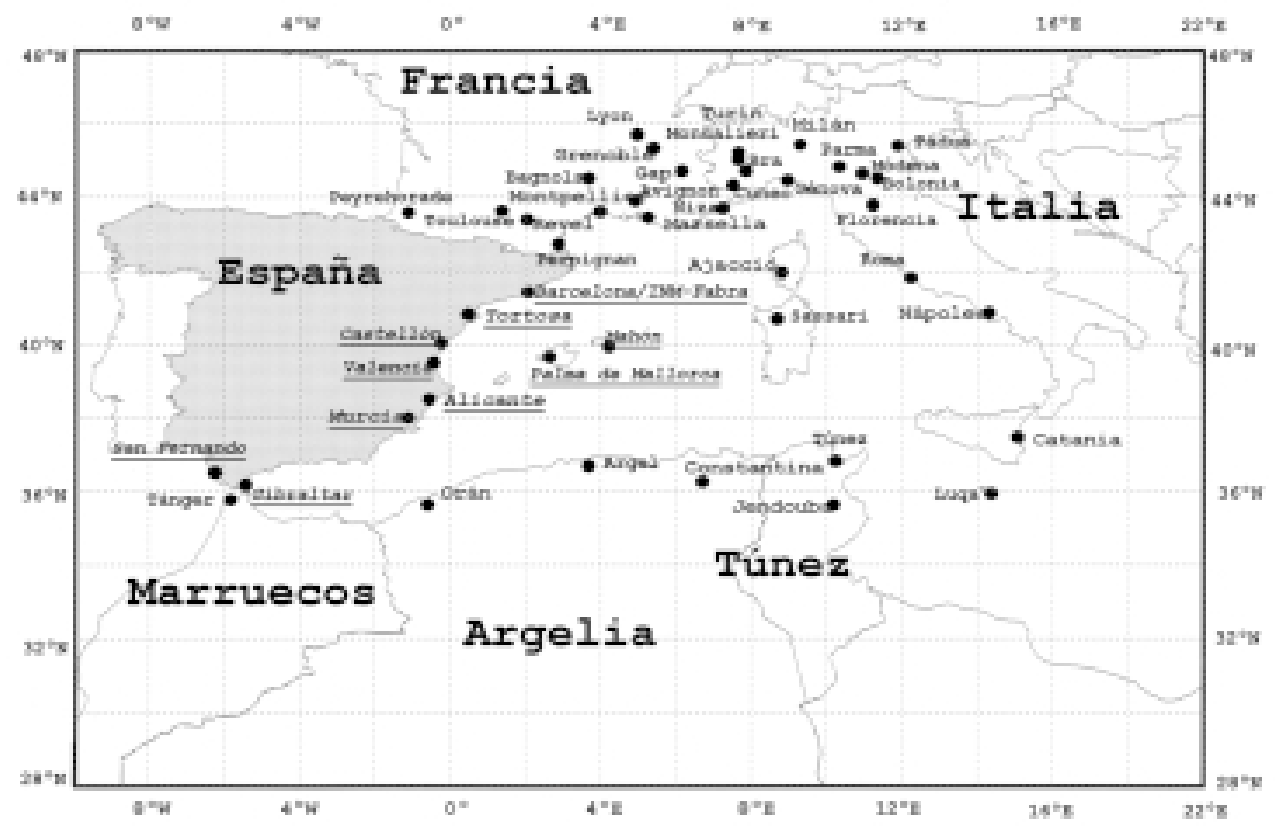

FIGURA 2. Localización de los observatorios históricos utilizados.

figura 2. Algunos de ellos cuentan con las series pluviométricas de mayor antigüedad mundial, tal es el caso de Pádua (1725) o el de Marsella (1749). La red regional de observatorios pluviométricos presenta así una malla aceptable y de suficiente longitud y continuidad. Las series tienen muy escasas lagunas de registros que han sido rellenadas utilizando el método de las correlaciones con observatorios próximos y de elevado coeficiente de correlación, $\mathrm{r}>0$ '7

Sin embargo, si bien todas estas series son aceptables en cuanto a duración y continuidad, no lo son tanto respecto a la posible homogeneidad de sus datos. Una gran parte de los observatorios que los han registrado han sufrido algún cambio de ubicación a lo largo de su historia. Ello ha podido originar la pérdida de homogeneidad de la serie obtenida. Es decir, que los datos manifiesten alguna tendencia de evolución no debida a causas o factores climáticos sino impuesta por los cambios de ubicación del observatorio. Unos cambios que bien conocidos en las series españolas, francesas y norteafricanas, ofrecen gran confusión en el caso de algunas series italianas.

En consecuencia, todas estas series pluviométricas han debido ser sometidas a un test de homogeneidad que acreditara su valor de fiabilidad para el análisis. Máxime en cuanto que, como hemos dicho, el «histórico» de algunos observatorios es desconocido o presentado contradictoriamente en las fuentes internacionales existentes (JONES, 1985; BRADLEY, 1985). El test de homogeneidad aplicado en todas las series es el de rachas, que complementariamente, en casos dudosos, ha sido contrastado con el de permanencias o de Helmert, así como los tests de Mann-Kendall y de Pettit. Ambos son los recomendados tanto por la O.M.M. como por el I.N.M. (O.M.M., 1990; NAYA, 1989).

La aplicación del test de homogeneidad ha mostrado que la mayor parte de las series analizadas respondían a una distribución aleatoria. Consecuentemente, estas series han 
sido consideradas aceptables para el análisis de la evolución pluviométrica. Sin embargo, algunas de las series analizadas (Tortosa, Alicante, Roma y Orán) han mostrado un valor de homogeneidad ajustado al límite de probabilidad aceptable ( $\mathrm{P} \leq 0$ '05). En todo caso, su reducido número (menos del 10\%) no resta validez a las conclusiones sobre la evolución pluviométrica regional obtenidas mediante el método de ajuste de regresión lineal aplicado a todos los observatorios.

\section{¿Qué muestra este análisis de evolución y tendencias pluviométricas en el Mediterrá- neo español?}

Los diez principales observatorios históricos que han venido registrando las precipitaciones mediterráneas españolas, no han escapado a estas dificultades. No obstante, su tratamiento permite obtener algunas conclusiones provisionales dignas de consideración.

La mejor síntesis de los resultados queda reflejada a través de las normales pluviométricas de cada serie, subdivididas en períodos de treinta años (Cuadro I).

Cuadro I

EVOLUCIÓN (mm) DE LAS MEDIAS PLUVIOMÉTRICAS POR PERÍODOS DE 30 AÑOS Y TENDENCIA

\begin{tabular}{|c|c|c|c|c|c|c|}
\hline Estación & $1840-1869$ & 1870-1899 & $1900-1929$ & 1930-1959 & 1960-1994 & Trends \\
\hline Barcelona (INM) & $563^{\prime} 95$ & $526^{\prime} 49$ & $576 ’ 05$ & $590 ’ 68$ & $584 ’ 90$ & $+0 ’ 45$ \\
\hline Barna (INM-Fabra) & $563 ’ 95$ & $526 ’ 49$ & $573 ’ 99$ & $597 ' 50$ & $649 ’ 11$ & +1 '03 \\
\hline Tortosa & & $532 ’ 13$ & 474’93 & $562 ’ 09$ & $557 ’ 22$ & $+0 ’ 45$ \\
\hline Castellón & & & $411^{\prime} 74$ & $420 ’ 36$ & $460 ’ 99$ & +0’91 \\
\hline Valencia & $501^{\prime} 79$ & $498^{\prime} 93$ & $416 ’ 27$ & 434’11 & $464 ’ 20$ & $-0 ’ 20$ \\
\hline Alicante & $418^{\prime} 32$ & $356^{\prime} 51$ & $349^{\prime} 73$ & $316 ’ 55$ & $352^{\prime} 00$ & $-0,34$ \\
\hline Murcia & $337^{\prime} 49$ & $378 ’ 34$ & $280 ’ 80$ & $300^{\prime} 13$ & $292 ’ 88$ & $-0 ’ 62$ \\
\hline Mahón & & $625^{\prime} 62$ & $615^{\prime} 22$ & $634^{\prime} 62$ & $578^{\prime} 51$ & $-0 ’ 53$ \\
\hline Palma & $362 ’ 09$ & $461 ’ 90$ & 483’95 & 464’32 & 417’24 & $-0,34$ \\
\hline Gibraltar & $880^{\prime} 71$ & $872 ’ 48$ & $867 ’ 24$ & 774’94 & 767’38 & $-1 ' 10$ \\
\hline S. Fernando & 653’37 & $707 ’ 40$ & $525^{\prime} 46$ & $576^{\prime} 01$ & 553’37 & -1'80 \\
\hline
\end{tabular}

Como muestra el cuadro I, la principal conclusión que puede extraerse es el gran contraste entre la evolución pluviométrica seguida por el sector mediterráneo septentrional de la Península Ibérica y la seguida por el resto del sector mediterráneo peninsular e insular español. Por un lado, el sector mediterráneo septentrional, observatorios de Barcelona (I.N.M. y Fabra), Tortosa y Castellón, muestran una evolución secular ascendente, especialmente marcada desde el último tercio del siglo XIX (Fig. 3).

En cambio, el resto de la región mediterránea española ha registrado una evolución contraria, descendente. En este sector central y meridional, la decadencia mostrada por las precipitaciones viene especialmente configurada por cuanto que la segunda mitad del siglo XIX fue sensiblemente más lluviosa que la media de todo el siglo XX. Un siglo, este último, que realmente no presenta tendencia alguna. Ello supone una contradicción estadística frente a las afirmaciones ampliamente sostenidas de una evolución regular descendente y frente a las previsiones de los actuales modelos de cambio climático (UNIPCC, 1995). 


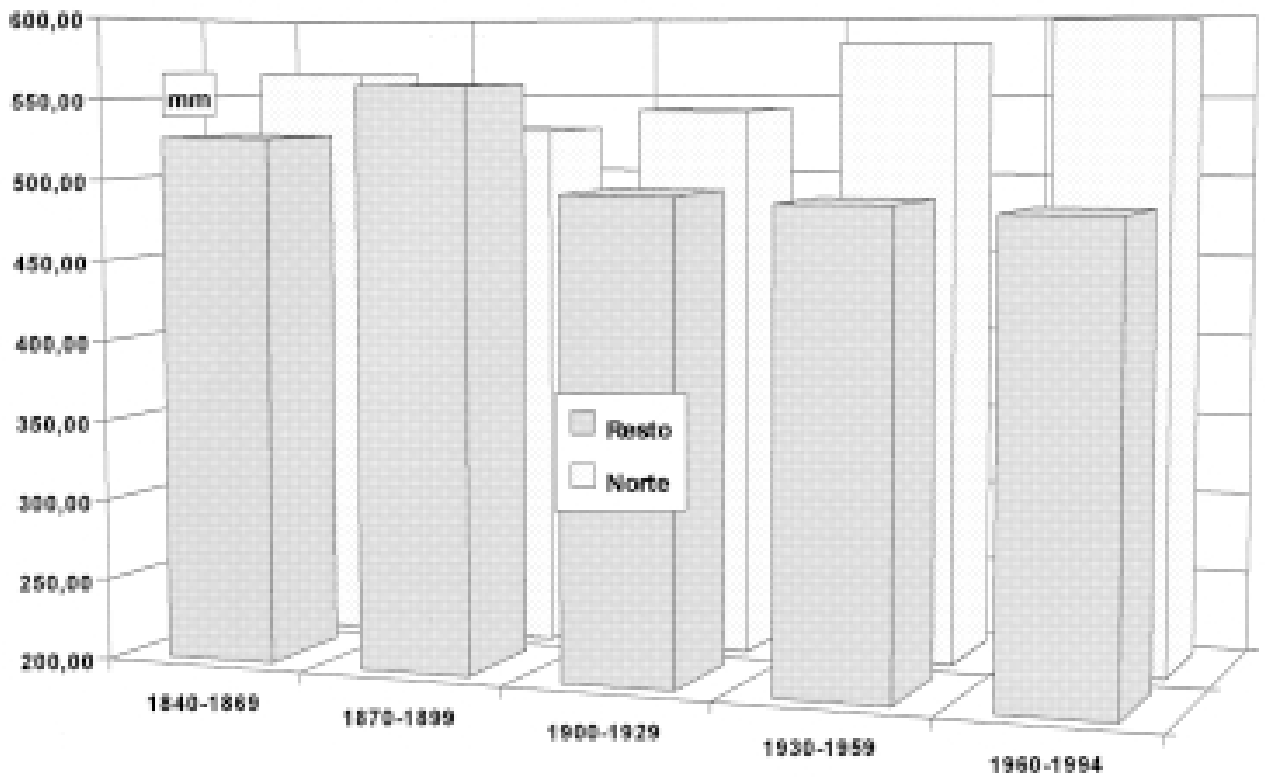

FIGURA 3. Comparación de la evolución de las medias pluviométricas en periodos de 30 años entre la zona al norte del paralelo 40 y los observatorios de la zona mediterránea sur.

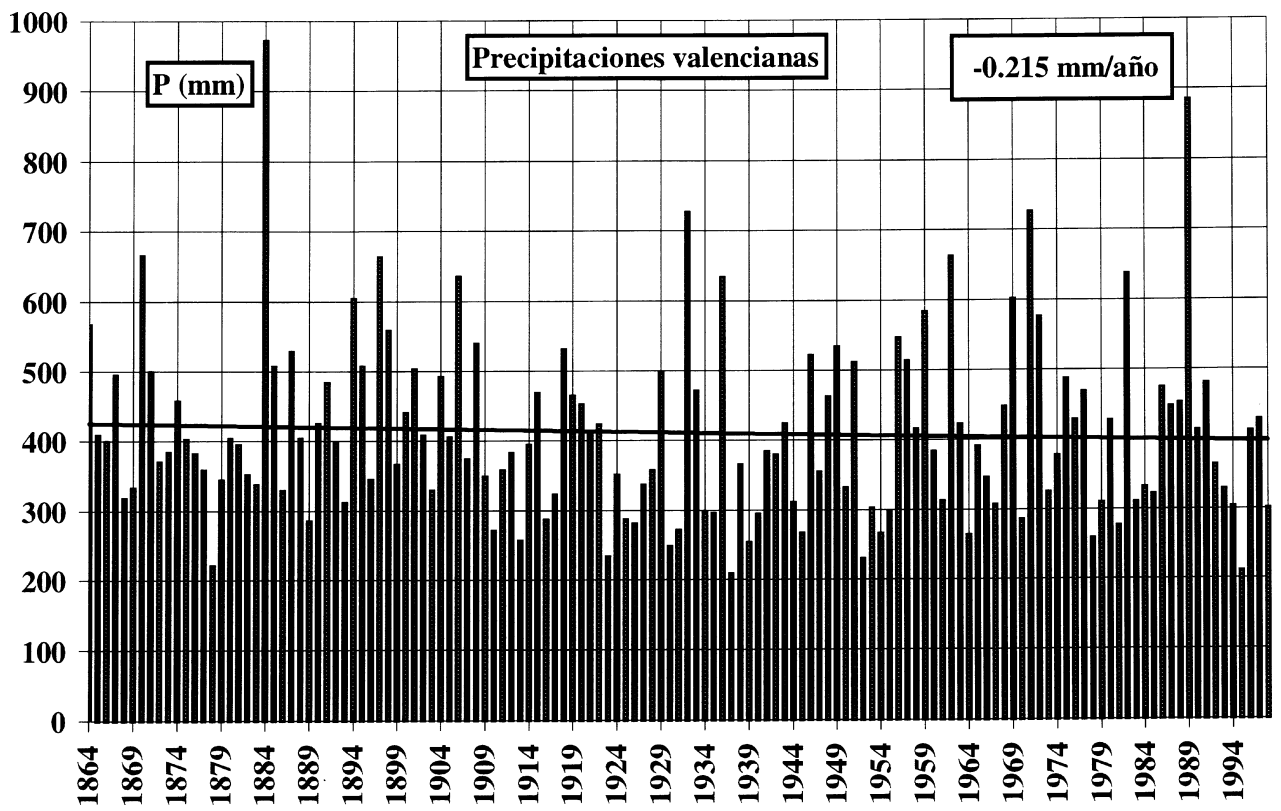

FIGURA 4. Evolución y tendencia de las precipitaciones medias valencianas (I.N.M.) 


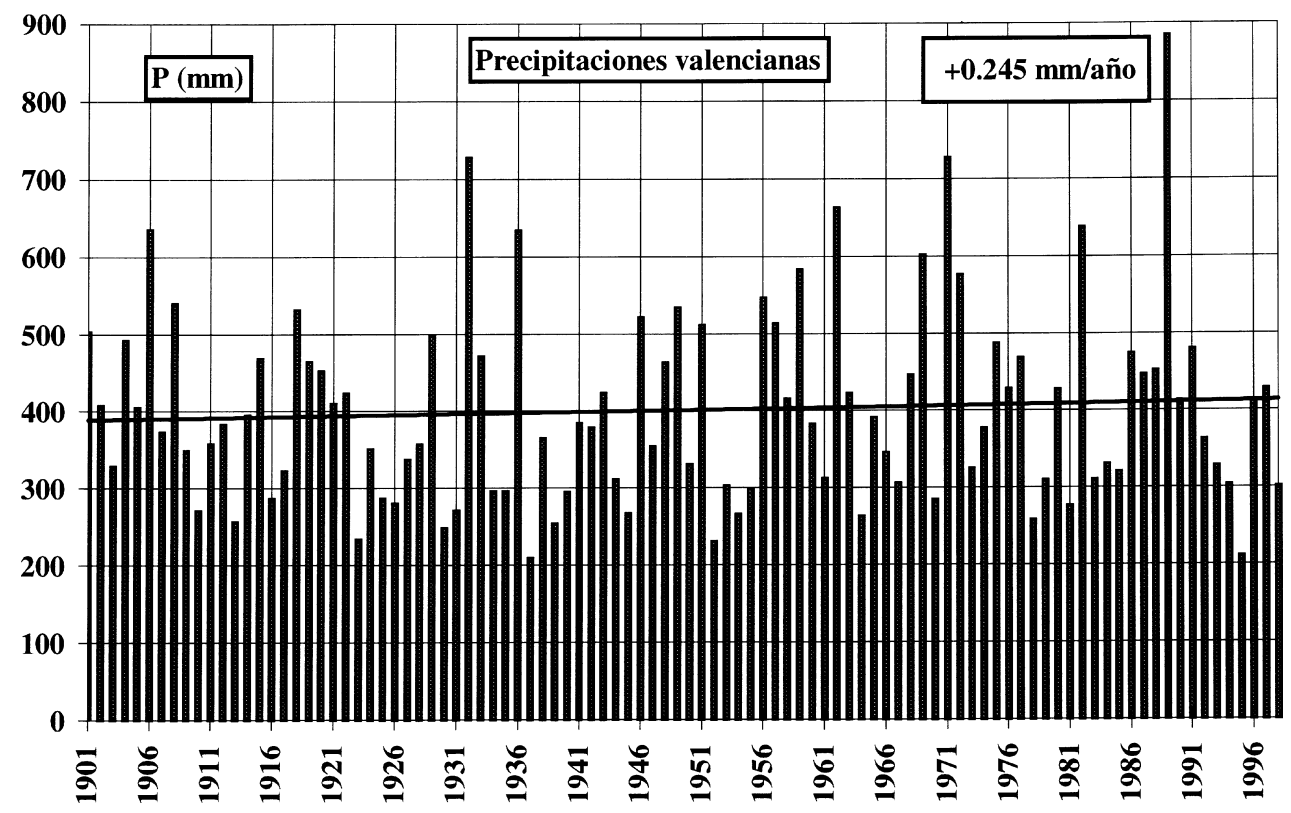

FIgURA 5. Evolución y tendencia de las precipitaciones valencianas a lo largo del siglo actual. El «trend» muestra que las precipitaciones medias habrían aumentado en unos $25 \mathrm{~mm}$ anuales (I.N.M.).

Máxime cuando los tests de Mann-Kendall y de Pettit no muestran valores de significación en las tendencias.

Este efecto puede observarse si las mismas series pluviométricas valencianas, globalmente con tendencia negativa desde mediados del siglo XIX (Fig. 4), las analizamos desde comienzos del siglo XX. La tendencia que manifiestan con un análisis a lo largo del siglo actual no parece corroborar la instauración de un escenario con mayor aridez (Fig. 5), sino, al contrario, de mayor humedad.

No obstante, es preciso observar que este cambio de signo viene «controlado» por el gran valor de las precipitaciones caidas en un solo año, 1989, el record histórico en las tierras valencianas y tan solo tras el de 1884, a la sombra de la erupción del Krakatoa. Si en la serie valenciana «ajustásemos» el valor de la precipitación de 1989 al valor de la media regional, $414 \mathrm{~mm}$, el resultado ya comenzaría a mostrar una tendencia nula. Siendo todo ello una muestra más de la cautela con que debemos de proceder en este tipo de análisis.

En síntesis, analizando globalmente las series desde mediados del siglo XIX, el «trend» secular de las precipitaciones en la región mediterránea española es positivo al norte del paralelo $40^{\circ} \mathrm{N}$. Desde esa latitud va intensificando su valor negativo cuanto más al sur. Ello supone que, en relación a la segunda mitad del siglo XIX, en buena parte de la región mediterránea, las actuales precipitaciones estarían siendo inferiores en cantidades de 20 a 186 milímetros al año (MORENO y MARTÍN VIDE, 1986), mientras las tierras septentrionales recibirían entre 46 y 103 milímetros más que en el siglo anterior.

No obstante, conviene reiterar que los test de tendencia no muestran valores significativos. Estos tests no hacen sino reflejar las complejas variaciones cíclicas que muestran las precipitaciones mediterráneas. Así, sobre la misma estabilidad de tendencia desde 1900, se inscribe la variación acontecida en los últimos sesenta años. La figura 6 permite apreciar, 


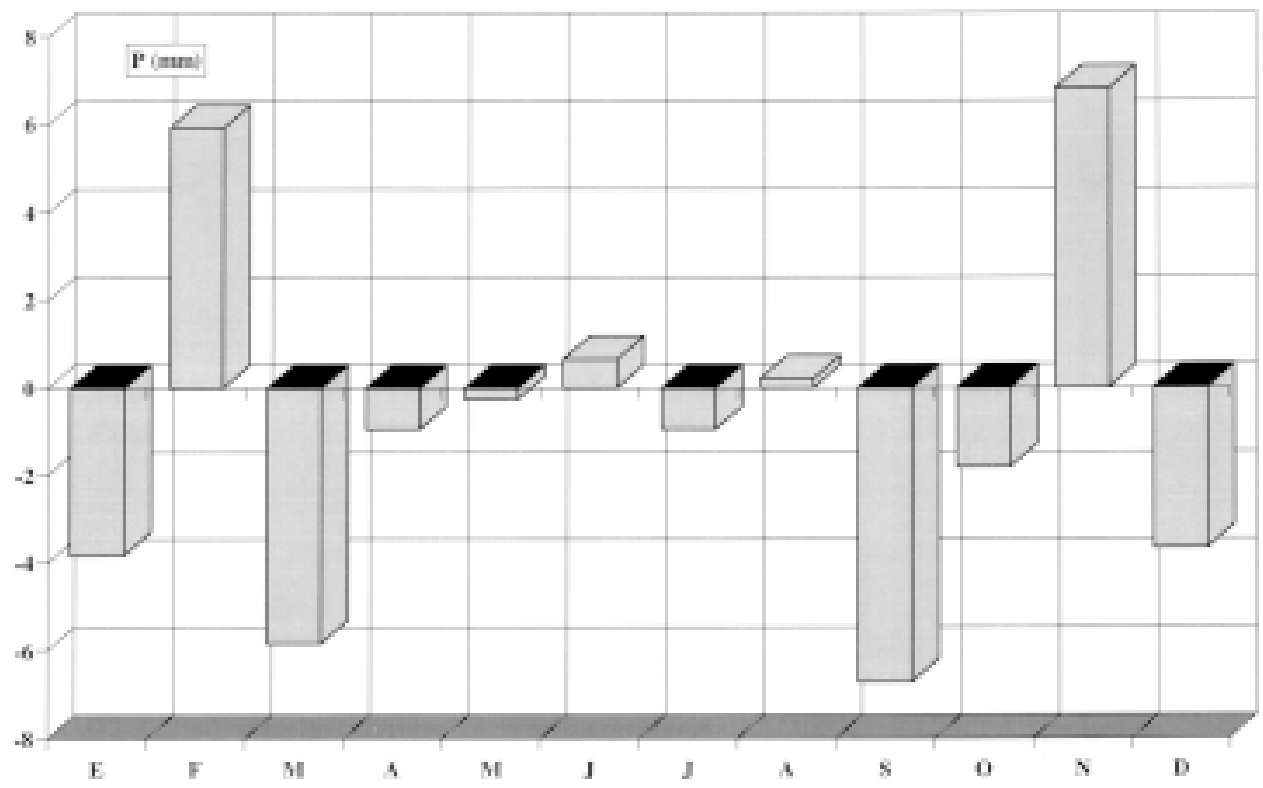

FigURA 6. Diferencias mensuales de precipitación entre los periodos 1961-1994 y 1931-1960. Fuente: I.N.M., Reial Acadèmia de Ciències i Arts, Meteorological Office y elaboración propia.
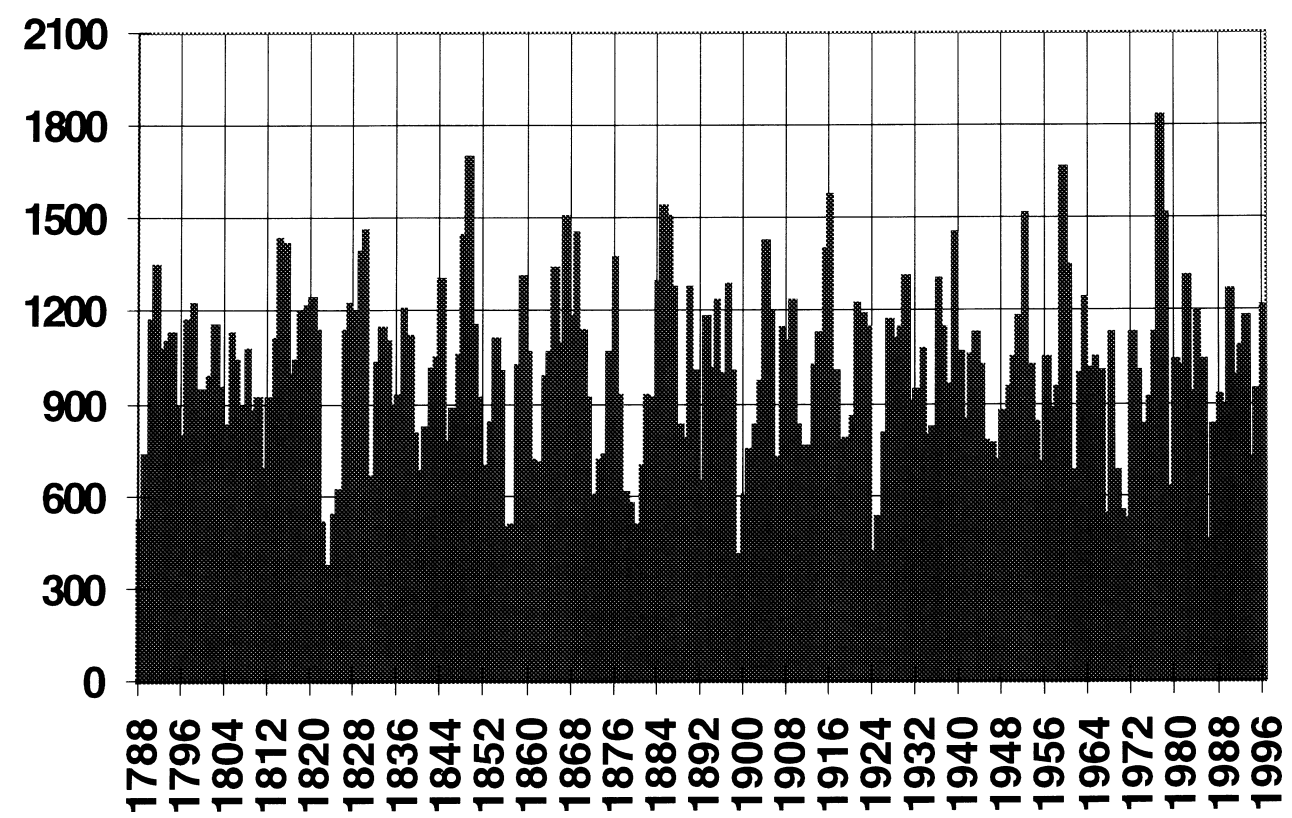

Figura 7. Cronología del monte Bobalar de Vistabella (Castellón) sobre Pinus nigra Arn. 
en el caso del Mediterráneo español, un pequeño cambio de régimen estacional durante los últimos sesenta años. Globalmente ha supuesto una reducción de las precipitaciones, equivalente a unos $10-12 \mathrm{~mm} / \mathrm{año}$.

Una hipótesis de estabilidad climática que asimismo ha podido ser reconstruida mediante el análisis dendroclimático de diversas zonas forestales. La figura 10 muestra el resultado de la reconstrucción realizada sobre un bosque de Pinus nigra en las vertientes del Pico de Penyagolosa, Bajo Maestrazgo. La cronología establecida mediante la técnica de estandarización y correlación no deja lugar a dudas. La evolución climática de las precipitaciones no parece mostrar tendencia significativa alguna basada en algún tipo de stress hídrico (Fig. 7).

\section{¿Qué muestra el comportamiento regional?}

Dada la unidad de la cubeta mediterránea, especialmente en cuanto a los factores geográficos y atmosféricos, cabe interrogarnos sobre el comportamiento regional de la pluviometría en aras de una mayor significación de las tendencias observadas en el Mediterráneo español.

La conclusión que desprende el análisis de las series concernientes a los siglos XIX y XX es que el Mediterráneo francés ha registrado un «trend» secular de crecimiento pluviométrico (MARTIN, 1984; DELANNOY, 1984). Este aumento de las precipitaciones es general en todos los observatorios analizados. (Fig. 8).

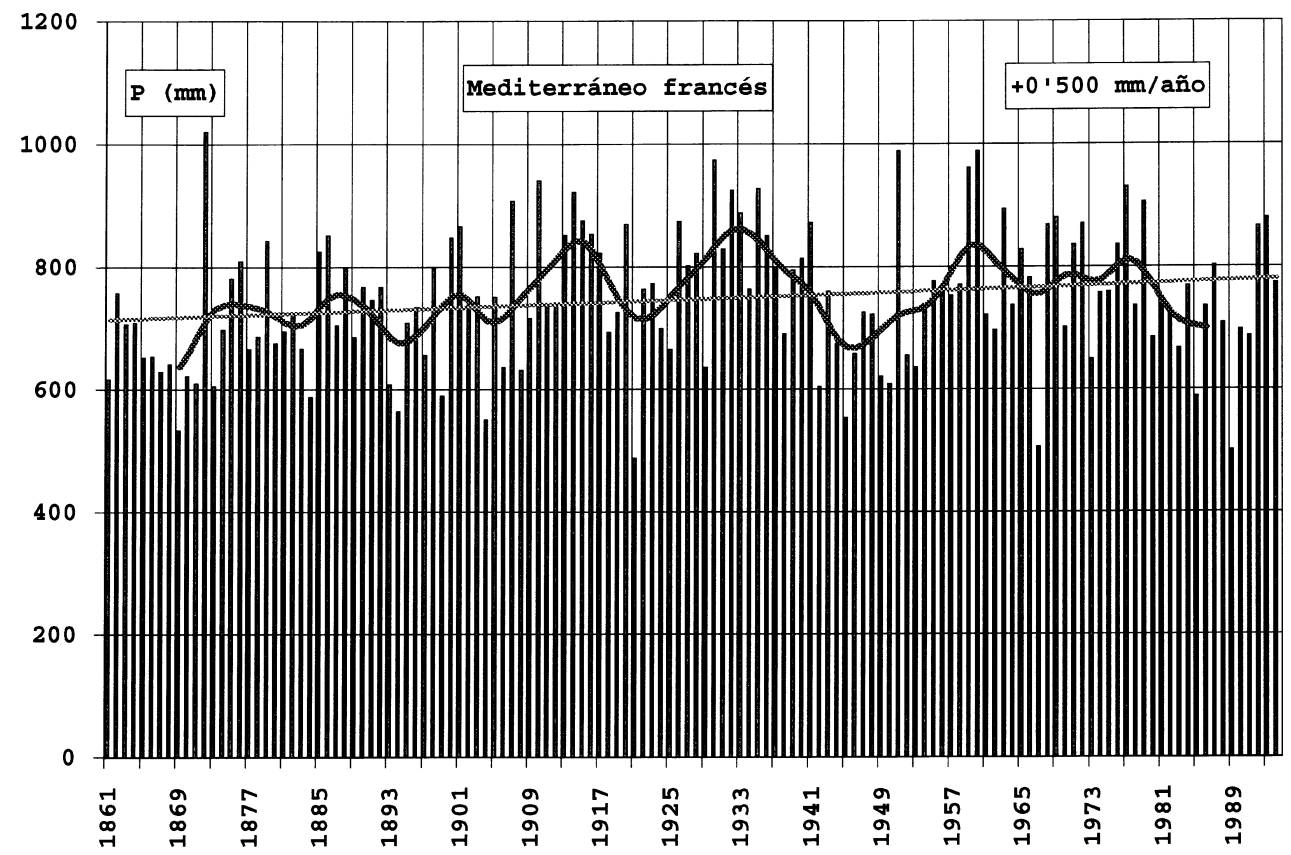

Figura 8. Evolución, tendencia y filtros de los totales anuales de precipitación global del Mediterráneo francés (1861-1994). Fuente: Meteo-France. 


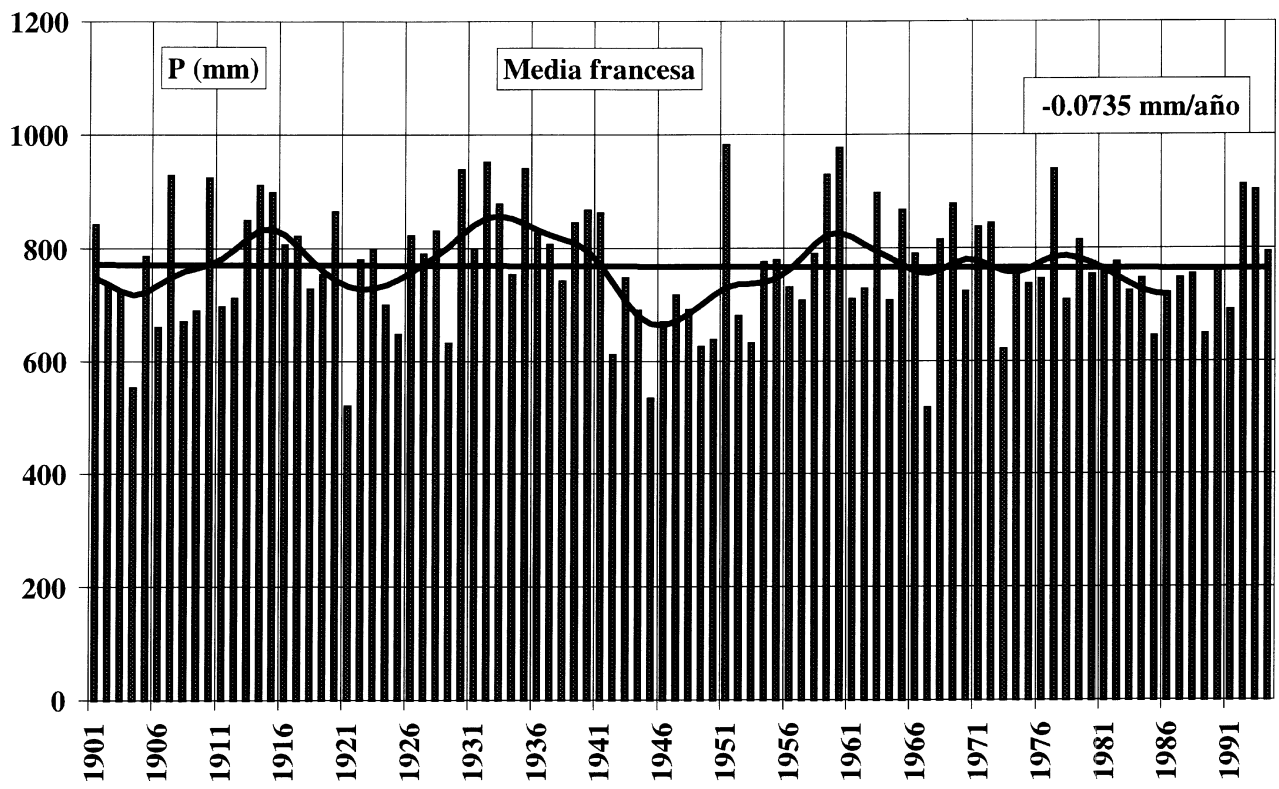

FIGURA 9. Evolución, tendencia y filtros de los totales anuales de precipitación global del Mediterráneo francés (1901-1994). Fuente: Meteo-France.

Esta conclusión adquiere un mayor interés por cuanto que viene a confirmar el comportamiento pluviométrico observado en la zona septentrional del Mediterráneo español. Una tendencia regional que parece reafirmada por el hecho de que los valores más ascendentes de «trend» pluviométrico secular se alcancen en los observatorios más septentrionales y ya en el el arco exterior del Mediterráneo: Grenoble y Lyon. En cambio, los valores de incremento pluviométrico son más débiles en los observatorios costeros o insulares: Marsella, Ajaccio, Montpellier y Perpiñán. Es más, al igual que en el caso español, si la tendencia global la analizamos a través del siglo XX, el «trend» carece de valor (Fig. 9).

No obstante, esta cohesión geográfica hispanofrancesa es rota por la evolución pluviométrica italiana. Las series italianas muestran un modelo predominante de disminución pluviométrica (Fig 10). Ello supone un comportamiento pluviométrico diferenciado con respecto a la región mediterránea septentrional española y mediterránea francesa.

Conviene, sin embargo, notar que este modelo de evolución pluviométrica negativa que muestran la mayor parte de los observatorios italianos no es absoluto. Así, frente a él, se opone el modelo configurado por las series de los observatorios de Bolonia, Bra, Parma y los de los observatorios meridionales de Nápoles y de Luqa, en la isla de Malta. Estas series manifiestan una evolución creciente de las precipitaciones con un «trend» secular especialmente acusado en el último tercio del siglo XX.

Curiosamente, esta tendencia de aumento pluviométrico registrada en los observatorios de Malta y Nápoles, enlaza con la mostrada en el norte de África por los observatorios de Argel y Túnez. En cambio, ya más hacia el extremo occidental del arco norteafricano, la evolución pluviométrica se caracteriza predominantemente por las tendencias hacia una reducción de las precipitaciones. Tendencias bien representadas en los observatorios de Constantina, Jendouba, Orán y Tánger (Cuadro II). 


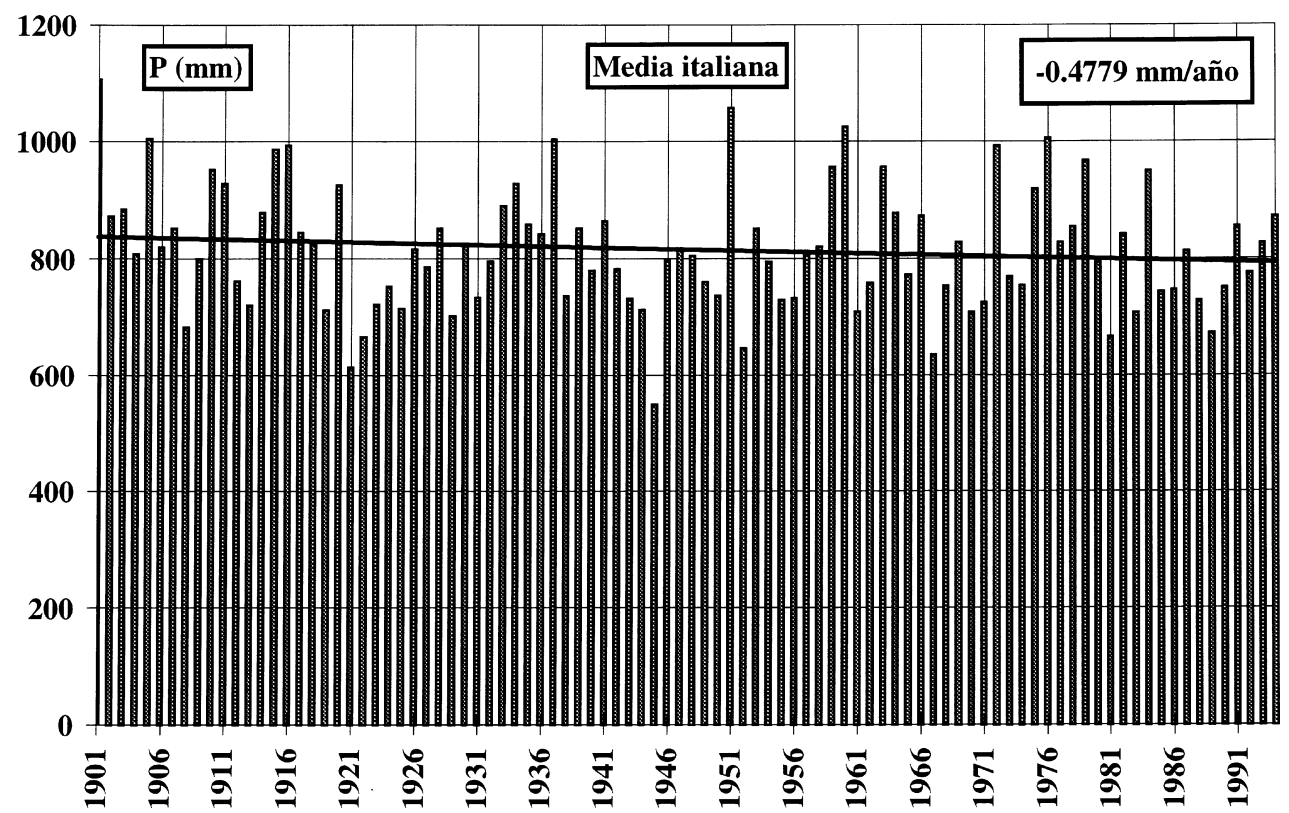

FIGURA 10: Evolución, tendencia y filtros de la serie media de precipitación de la Italia mediterránea (1901-1994).

Cuadro II

EVOLUCIÓN (mm) DE LAS MEDIAS PLUVIOMÉTRICAS NORTEAFRICANAS POR PERÍODOS DE 30 AÑOS Y TENDENCIA DESDE 1861

\begin{tabular}{|c|c|c|c|c|c|c|}
\hline & $1800-49$ & 1850-99 & 1900-29 & 1930-59 & 1960-94 & trend \\
\hline ARGEL & & $627^{\prime} 80$ & $621 ' 83$ & $681 ' 50$ & 673'91 & $+0 ’ 4883$ \\
\hline CONSTANTINA & $734^{\prime} 20$ & $586 ’ 34$ & $570 ’ 33$ & $519 ’ 98$ & $508^{\prime} 75$ & $-0 ’ 6429$ \\
\hline JENDOUBA & & & $451^{\prime} 03$ & $477^{\prime} 83$ & $447^{\prime} 31$ & $-0 ’ 0391$ \\
\hline ORÁN & $460 ’ 84$ & $518 ’ 49$ & $375^{\prime} 28$ & $396 ’ 97$ & $361^{\prime} 95$ & $-1 ’ 9628$ \\
\hline TÁNGER & & $812 ’ 59$ & $774^{\prime} 08$ & $856^{\prime} 48$ & $765^{\prime} 07$ & $-0,5970$ \\
\hline TÚNEZ & & $426^{\prime} 46$ & $409 ' 16$ & $475^{\prime} 03$ & $463^{\prime} 67$ & $+0 ’ 7238$ \\
\hline
\end{tabular}

En consecuencia, tanto la falta de coincidencia en el comportamiento regional como las tendencias sostenidas durante el último siglo en la mayor parte de la Cuenca mediterránea, autorizan, en el estado actual de nuestros conocimientos y mientras prosiguen las investigaciones, a no rechazar la hipótesis de la estabilidad climática. Hipótesis que sostiene la persistente alternancia histórica de sequías y períodos húmedos de mayores precipitaciones. Unas observaciones que nos sitúan ante una nueva reflexión.

\section{¿Una evolución cíclica?}

Como hemos visto, en la región mediterránea española, los análisis pluviométricos y dendroclimáticos se encargan de mostrar la clara alternancia de períodos secos y períodos 
húmedos. Es decir, toda una serie de variaciones más o menos rítmicas que sugieren una posible relación con procesos físicos.

De este modo, resulta evidente la importancia que tiene el ahondar al máximo en la búsqueda de posibles ciclos climáticos que ayuden al hombre a prevenir esos fuertes condicionamientos naturales como la sequía actual. Contrariedades que creíamos haber superado con la euforia del progreso técnico en que el medio parecía dominado por el hombre. Hoy, más que nunca, es preciso volver a interrogarnos: ¿Está el clima sujeto a ciclos? ¿Hasta dónde es posible llevar el comportamiento aparentemente errático de las precipitaciones del clima mediterráneo?

Si bien las curvas gráficas de los totales pluviométricos analizadas, nos muestran la característica dispersión en dientes de sierra, la búsqueda de una distribución más regular y previsible nos ha llevado a intentar la traza de una evolución cíclica. El procedimiento matemático ha consistido en combinar el producto de las medias variables y los pesos o valores binomiales de cada precipitación anual (J.M. MITCHELL et al., 1966).

Un filtro simétrico se puede expresar matemáticamente por la ecuación:

$$
\mathrm{x}_{\mathrm{t}}=\Sigma \omega_{\mathrm{i}} \mathrm{x}_{\mathrm{t}+\mathrm{i}}
$$

en la que $\mathrm{x}_{\mathrm{t}}$ es el valor filtrado de la serie temporal correspondiente al t-ésimo término y donde los pesos $\omega_{i}$ que afectan a los sucesivos términos de la serie varían simétricamente a derecha e izquierda de un peso central. El filtro definido de esta forma tiene una longitud de $2 \mathrm{n}+1$ unidades de tiempo.

El filtro empleado en este estudio se ha obtenido tomando un conjunto de pesos $\omega_{\text {i }}$ proporcionales a los coeficientes binomiales.

$$
C_{k}=\frac{n !}{k !(n-k) !}
$$

El factor de proporcionalidad está determinado por el hecho de que la suma de los pesos que intervienen en la ecuación (1), sea igual a la unidad. Esto es,

$$
\Sigma \omega_{\mathrm{i}}=1
$$

El factor $\mathrm{n}$ se elije de forma que la longitud de onda $\lambda$ sea igual a 6 veces la desviación estandard $\sigma$

$$
\lambda=6 \sigma=3 \sqrt{ } \mathrm{n}
$$

en la práctica se toma el entero par más próximo al valor determinado por esta última ecuación.

Con este tratamiento estadístico puede adoptarse cualquier longitud de onda $\lambda$. La elección de este valor no debía ser arbitraria sino relacionada con algún proceso atmosférico ó astronómico. Siendo que todas las estructuras atmosféricas parecen seguir las migraciones solares de latitud, no parece erróneo suponer que los ciclos climáticos pueden modularse sobre la actividad energética solar. Así, durante los últimos cuatro siglos, los años meteorológicos excepcionales para las vendimias francesas confirman su relación con la actividad solar e incluso la destacan mejor (J.P. LEGRAND, 1978).

El sol presenta una actividad energética que se manifiesta, en particular, por la aparición de grupos de manchas sombreadas sobre la superficie solar, desplazándose como 
consecuencia de su rotación en 27 días. Aunque esto es conocido desde el siglo XVII, el índice de actividad solar fue propuesto por Wolf en 1868. Descansa en la observación de las manchas solares y sigue siendo el empleado por los observatorios astronómicos actuales.

$$
R=K(10 g+f)
$$

siendo $\mathrm{K}$ un coeficiente del observatorio; $\mathrm{f}$, el número de manchas visibles en la superficie solar; g, número de grupos distintos.

Siendo que todas las estructuras atmosféricas parecen seguir las migraciones solares de latitud, no parece erróneo suponer que los ciclos climáticos puedan modularse sobre la actividad energética solar. Consecuentemente, la búsqueda de estas relaciones se ha basado en las variaciones de la actividad solar. Unas variaciones manifestadas por los ciclos medios undecenales de las manchas solares y evaluadas mediante el índice o número de Wolf.

Globalmente puede considerarse que la evolución pluviométrica regional muestra una buena correspondencia con la actividad solar. En la configuración de las dos curvas podemos observar que los ciclos pluviométricos y los ciclos solares presentan un aceptable $\mathrm{o}$, al menos, sugestivo paralelismo. Es decir, a lo largo de los once ciclos modulados entre 1866 y 1994, una buena parte de los máximos y mínimos solares, preceden con uno o dos años de decalado a los máximos y a los mínimos pluviométricos (Fig. 11).

No obstante, esta correlación no es perfecta. Su valor no supera un $75 \%$ de probabilidad. Especialmente podemos observar que existen dos grandes excepciones en esta configuración. Estas son las correspondientes a los dos máximos solares de 1938 y de 1980 que coinciden con un mínimo pluviométrico. En 1980, la explicación satisfactoria que podemos encontrar es el efecto de la actividad volcánica del Santa Helena (1980) y del Chichón (1982), cuyos aerosoles han podido bloquear la mayor radiación solar y su potenciación de

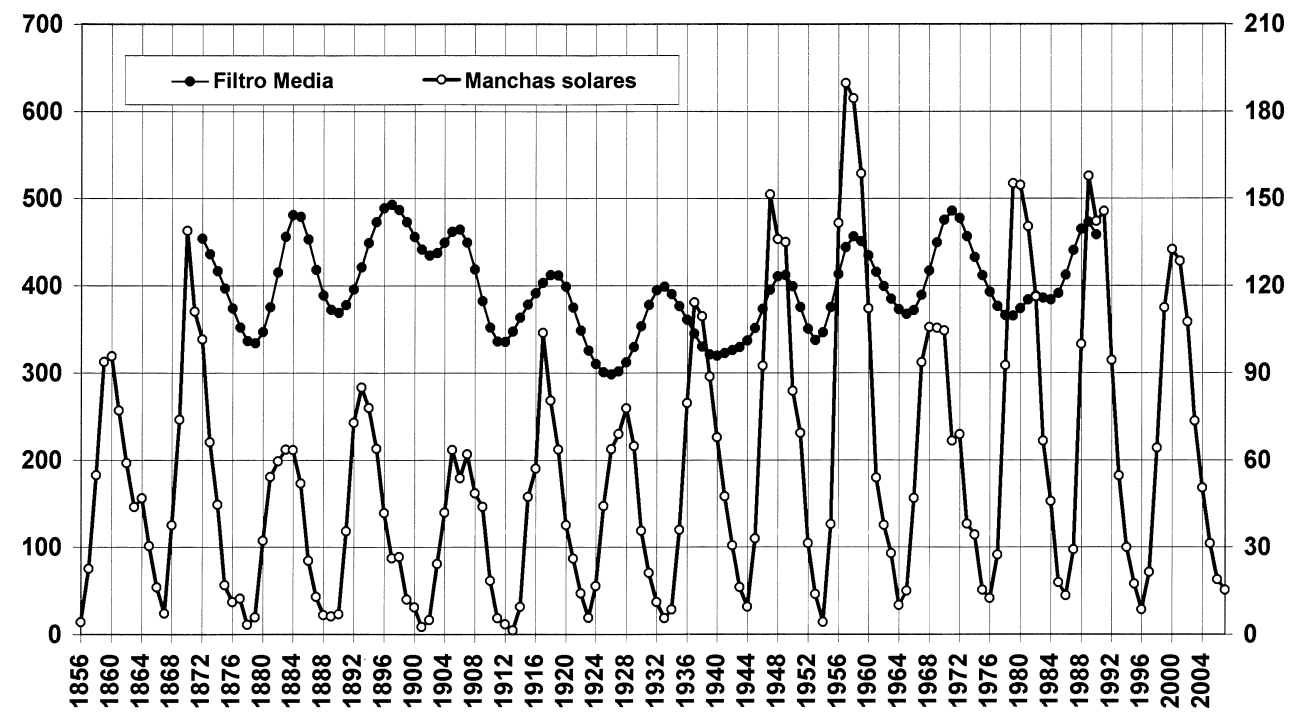

Figura 11. Evolución de los ciclos solares y de los pluviométricos filtrados. 
mayores precipitaciones. Éstas sólo se recobran ya en el mismo ciclo actual con su máximo en 1989 y 1990 y su mínimo en 1995-1996, paralelamente a la caída solar y que, afortunadamente esperemos, ha comenzado a recobrarse con su proyección de máximo solar en el año 2000.

\section{Factores físicos}

Ese sugestivo paralelismo de las curvas pluviométricas filtradas y los ciclos solares bien podría estar basado en alguna relación física más o menos compleja, esencialmente sobre dos procesos: las propias variaciones de intensidad en la irradiación solar y las interacciones atmósfera-mar.

\section{La radiación solar}

El papel decisivo de estos ciclos de variaciones de la energía solar no debe parecer simplista. En la naturaleza, cualquiera que ésta sea, cósmica o terrestre, todo se rige por lo térmico. Es por ello por lo que en la superficie de la tierra, los movimientos atmosféricos y oceánicos no escapan a esta regla. Ellos son todos el resultado de contrastes de temperatura más o menos acusados entre las diferentes zonas del planeta.

Factor por excelencia de esos contrastes térmicos son las variaciones de la radiación solar. Actualmente, los registros del satélite Nimbus permiten observar bien estos ciclos de radiación y su correspondencia con los ciclos solares definidos por el número de Wolf relativo a las manchas. Un paralelismo que permite observar igualmente dos rasgos importantes. El primero es que el máximo de energía parece sufrir un pequeño decalado anual con relación al máximo definido por el índice de Wolf (1989-1990). El segundo rasgo es el evaluar la diferencia de $2 \mathrm{~W} / \mathrm{m}^{2}$ entre máximos y mínimos de energía (Fig. 12).

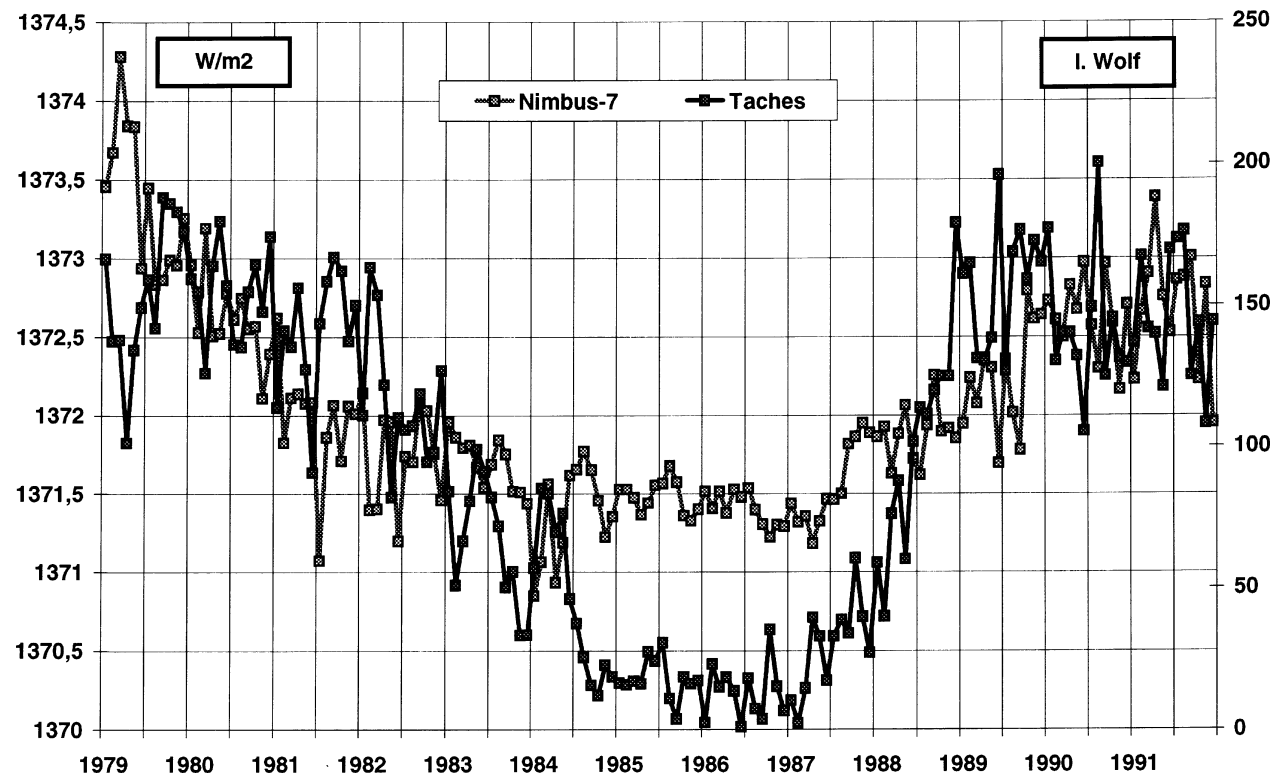

Figura 12. Registros de la Radiación Solar del satélite Nimbus y ciclo de manchas a través del número de Wolf. 


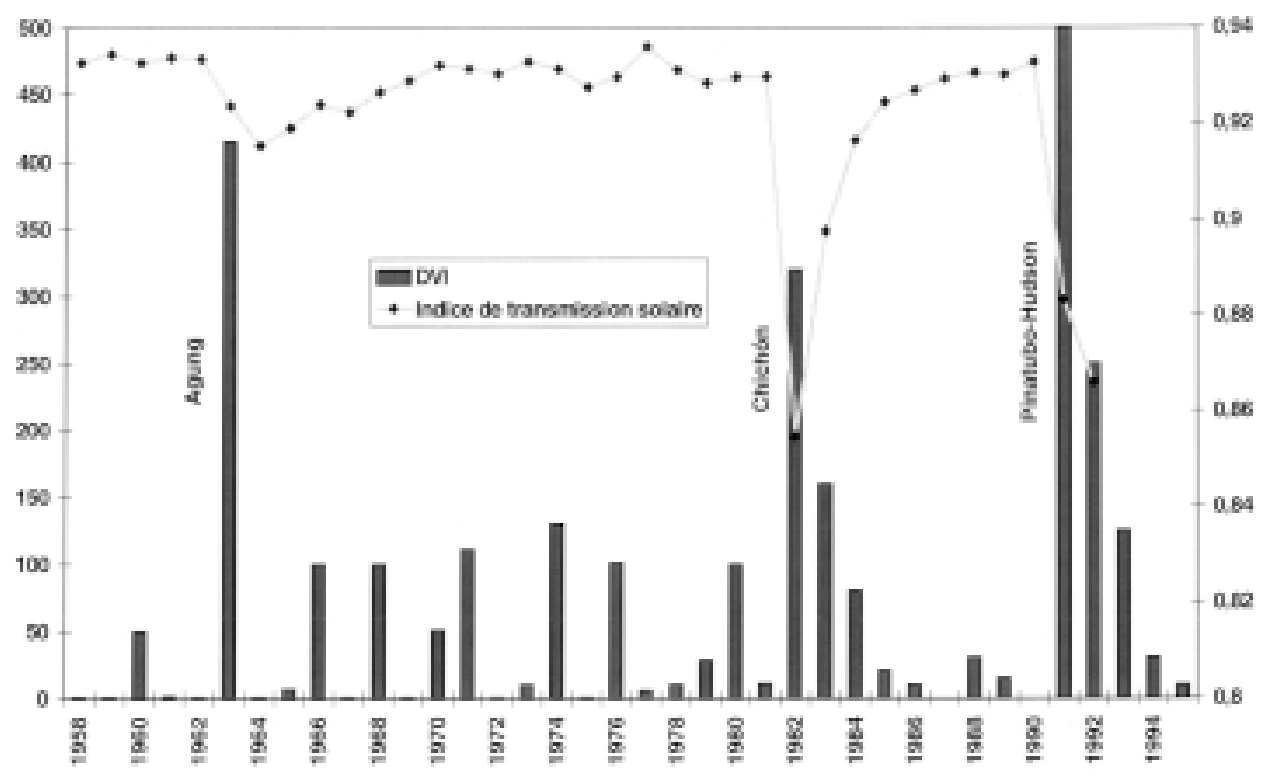

FIGURA 13. Medidas de transparencia atmosférica en el observatorio del Mauna Loa (E.G. DUTTON, 1994).

No obstante, como hemos dicho, la intensidad de la radiación recibida en superficie puede ser paliada, total o parcialmente por factores como la variación del albedo y de la transparencia atmosférica. En este sentido, los registros del observatorio del Mauna Loa desde 1958 permiten explicar algunas de las irregularidades entre los últimos ciclos solares y los ciclos pluviométricos. Tales serían la extensión del mínimo pluviométrico centrado en 1966, a la sombra del enfriamiento causado por el aerosol de l'Agung, y el abortamiento del máximo pluviométrico que debería haber correspondido al máximo solar de 1980. Las erupciones del Santa Helena (1980) y, en particular, del Chichón (1982), bien habrían podido suponer una anulación parcial en superficie del máximo solar observado sobre las manchas entre 1979 y 1980 y consiguientemente del máximo pluviométrico (Fig. 13).

En consecuencia, el «forcing» volcánico podría alterar la buena correlación entre ciclos pluviométricos y ciclos solares. Incluso el único ciclo anómalo que persiste, el mínimo pluviométrico coincidente con el máximo solar de 1937-1938, podría haber sufrido la acción volcánica de las islas Santorín, con pequeño DVI pero con gran efectividad en la región mediterránea. Su mejor reflejo es la notable caída de las temperaturas entre los años 1939 y 1940 que ha constituido el período más frío de toda la segunda mitad del siglo XX.

\section{Las interacciones atmósfera-mar}

El segundo fundamento físico de estas relaciones entre los ciclos pluviométricos y los ciclos solares puede estar constitutido por la acumulación de energía en el seno de las aguas marinas. Estas aguas y sus interacciones con la atmósfera juegan un papel casi decisivo en la Climatología de la Cuenca Occidental del Mediterráneo (J.M. JANSA,1951). Desde el fondo de esa cubeta orográfica, en función de sus temperaturas cálidas, su gran 
calor específico, su convección y facilidad de evaporación, la masa marina tiene una gran influencia en los procesos climáticos.

Este decisivo papel del campo térmico marino parece responder a uno de los principales hechos o rasgos físicos del océano, su alta capacidad calorífica, muy superior a la del aire. La energía térmica contenida en un litro de agua corresponde a la contenida en un volumen de tres metros cúbicos de aire a la misma temperatura. El mar, que cubre el $71 \%$ del planeta, constituye así el mas formidable de los reservorios de calorias. Ello, unido a su gran capacidad conductiva, determina asimismo su alta inercia térmica. Esta es 1.200 veces mayor que la de la atmósfera.

En todos estos procesos, los mecanismos que intervienen en la estructura térmica vertical del mar Mediterráneo, adquieren una significación especial. La formación del campo térmico superficial, a lo largo de las diferentes estaciones del año, obedece a diferentes mecanismos físicos. Así, durante el invierno, la temperatura superficial del mar suele ser más elevada que la del aire. Esta diferencia determina una intensa emisión de calor desde el mar hacia la atmósfera y consecuentemente un enfriamiento de las aguas superficiales. A su vez, este enfriamiento engendra una activa convección libre en el seno de las aguas marinas con la profundización o desaparición sensible de la termoclina. La subsidencia de las aguas más frías de superficie contribuye así a crear un gran espesor de aguas homotermas en el nivel superior de las aguas marinas. Espesor que alcanza su máximo valor a finales del invierno y que en esta estación proporciona el máximo volumen de capa activa implicada en el intercambio de calor con la atmósfera (Fig. 14).

Durante el verano, por el contrario, la intensa radiación solar y altas temperaturas ambientales calientan las capas superficiales del mar limitando la turbulencia convectiva. La termoclina aparece muy en superficie y la capa activa implicada en los intercambios con la atmósfera se torna muy delgada. Simultáneamente, esta capa activa en los intercambios de calor con la atmósfera aparece separada del agua subyacente por la termoclina.

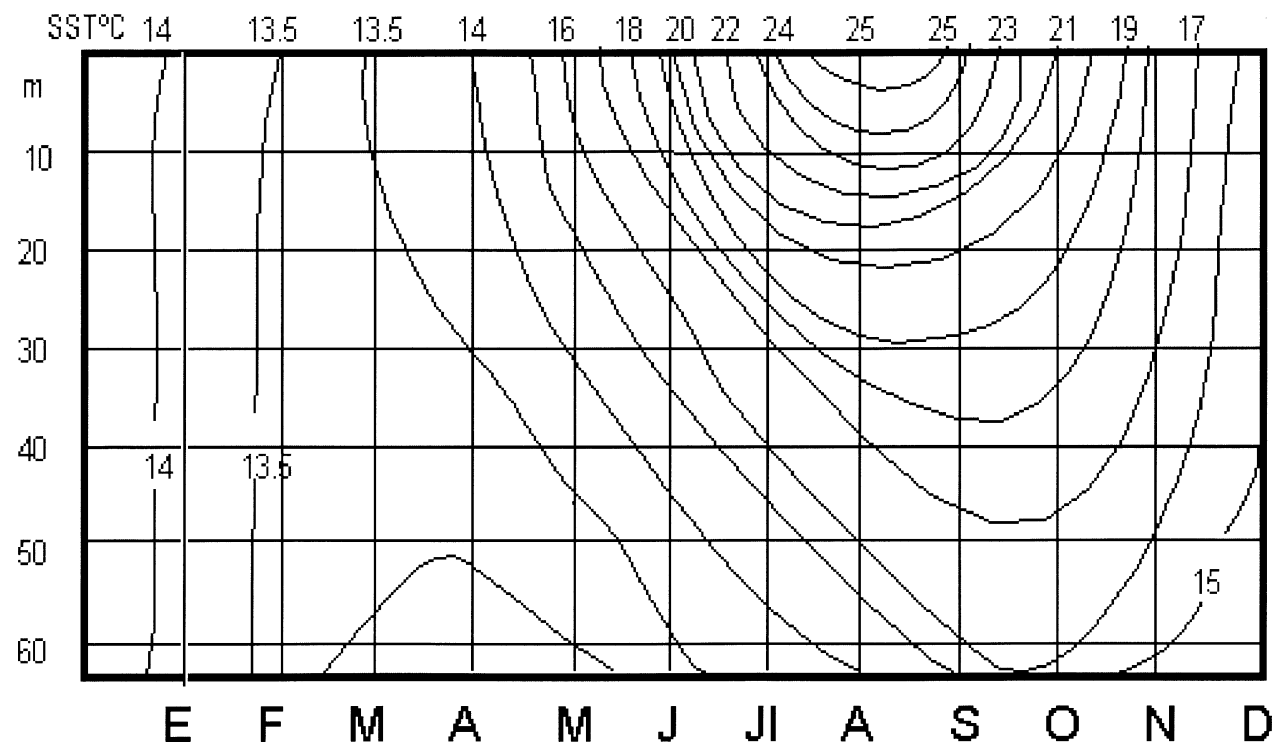

FIGURA 14. Isotermas en profundidad de las aguas mediterráneas y formación de termoclina. 


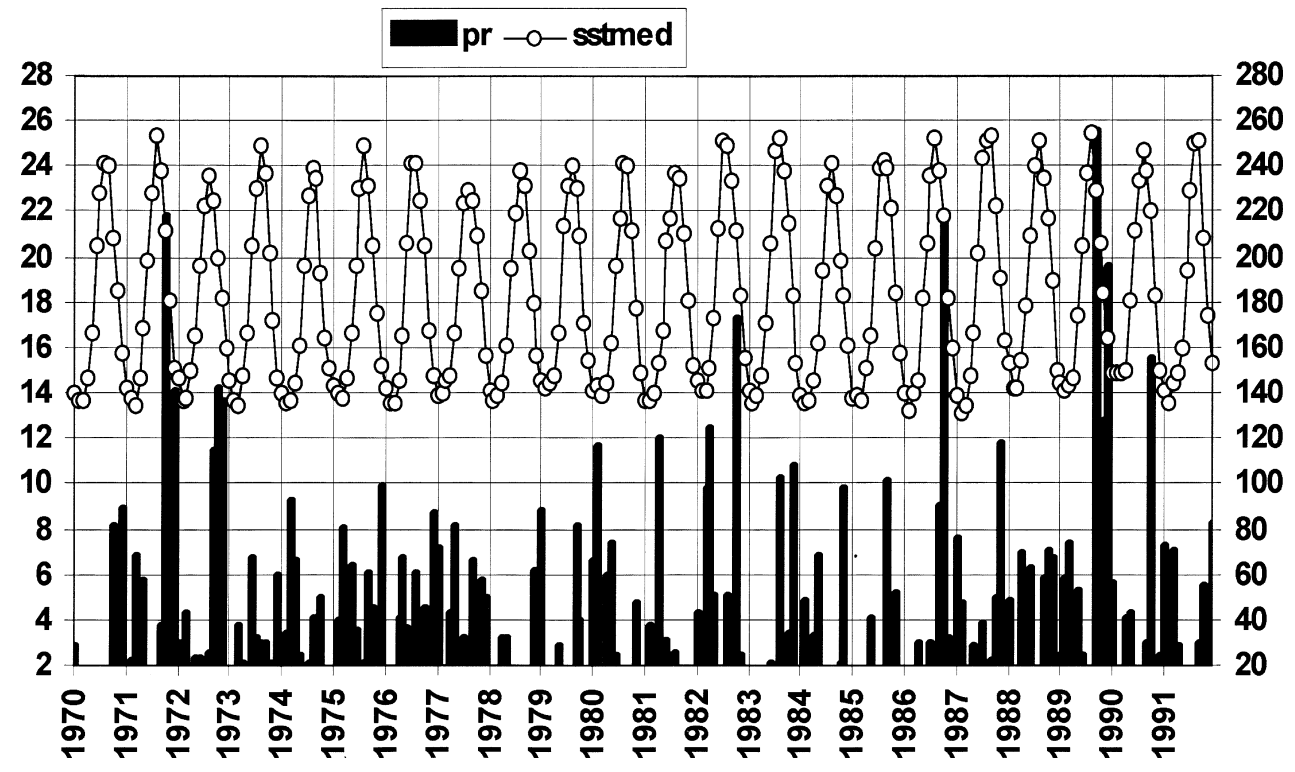

FIGURA 15. Relación de las grandes ciclogénesis mediterráneas con las anomalías positivas de la temperatura superficial del mar

De todos estos procesos se puede deducir que los intercambios de calor entre mar y atmósfera deben ser muy débiles en la época estival a causa del menor espesor de la capa activa del océano implicada en las interacciones. Consiguientemente se acepta que, durante el verano, el mar realiza, casi exclusivamente, una acumulación de calor. Es un proceso que muestra grandes oscilaciones ya que las variaciones en las anomalías térmicas superficiales de los océanos Atlántico Norte y Pacífico Norte y del mar Mediterráneo han mostrado que su máximo de variabilidad acontece en la estación cálida, entre julio y septiembre.

De este modo, el calentamiento estival de la masa marina es un proceso clave para la climatología mediterránea. Los elevados valores que la temperatura superficial del mar puede alcanzar a fines del verano se muestran particularmente críticos. Esas exageraciones de gradiente entre una masa marina más cálida, sobre los $26^{\circ} \mathrm{C}$, y las masas aéreas más frías pueden desencadenar toda la convección potencial del mar. No es otra la génesis de los grandes procesos ciclogenéticos de fin de verano y de otoño, cuando la inercia térmica del mar mantiene todavía sus temperaturas elevadas y las masas de aire polar comienzan sus primeras irrupciones hacia las latitudes meridionales, tal vez drenadas para equilibrar esa anomalía positiva de las temperaturas marinas (Fig. 15). Unas anomalías positivas que están básicamente controladas por la energía solar incidente sobre el mar y consecuentemente con los máximos del ciclo solar.

\section{Conclusiones}

La enorme inquietud provocada por la escasez actual de recursos hídricos y la dramática imagen que ello representa para la región mediterránea, implica la necesidad de analizar rigurosamente la evolución pluviométrica. Dentro de este análisis una cuestión 
clave se plantea: ¿Estamos asistiendo a una reducción progresiva de nuestras precipitaciones? Una interrogante a la que los análisis realizados no permiten dar una respuesta afirmativa. Es más, los resultados permiten sostener la hipótesis de una estabilidad climática.

No obstante, esta misma hipótesis de la estabilidad climática supone la existencia de la ancestral sucesión de períodos secos y humedos típica del clima mediterráneo. Observación que, ante las crecientes necesidades de recursos hídricos, obliga a un nuevo interrogante: ¿Está nuestro clima sujeto a ciclos? Una cuestión a la que los ciclos de actividad solar modulados por las resonancias convectivas podrían aportar respuestas interesantes.

Finalmente cabe replantearse una respuesta a la motivación de las interrogantes anteriores. Es muy posible que la verdadera razón de las angustias que los ciclos secos provocan actualmente no es otra que su proyección sobre unos consumos que se han situado ya sobre el nivel de las precipitaciones normales. De ahí que cuando éstas no se producen las señales de alerta se disparan. Sin duda que las sequías de principios de siglo no causaban tanta alarma. Baste recordar que la dotación urbana de esos años era tan sólo de 20 litros por habitante y día, mientras que actualmente nuestra «calidad de vida» precisa unas dotaciones de 400 litros por habitante y día. Tales son los aumentos de una demanda urbana que se añaden a las necesidades industriales y muy especialmente a las agrícolas que suponen más del $75 \%$ de la demanda total de agua. Su magnitud permite calcular bien los aumentos de nuestro consumo conociendo que la extensión regada en España a principios de siglo era de 1.200.000 Has. mientras que actualmente se ha multiplicado por tres, ascendiendo a una cifra próxima a los 3.500.000 Has. (MORALES GIL, A., 1999).

\section{Bibliografía}

BIANCOTTI, A. y MERCALLI, L. (1991): «Variazioni climatiche nell'Italia nord-occidentale». Memorie della Società Geografica Italiana, Vol. XLVI. pp. 385-408.

BRADLEY, R. S. et alters (1985): Climatic Data Bank for Northern Hemisphere Land Areas. 1851 1980. TR017, DOE/EV/10739-2, CDIAC, U.S. DOE.

CAMUFFO, D., BERNARDI, A. y ONGARO, A. (1991): «Le variazioni recenti del clima (18001990) e le prospettive per il XXI secolo». Memorie della Società Geografica Italiana, Vol. XLVI. pp. 363-384.

CDIAC (1994): «Trends'93». Oak Ridge National Laboratory, Carbon Dioxide Analysis Information Centre.

DARCHEN, J. (1989): «Influence des mers et des oceans». Met-mar, 145.

DELANNOY, H. et DOUGUEDROIT, A. (1984): «Les variations des précipitations printanières dans le sud-ouest européen et le Maghreb occidental (1916-1965)». Revue Géographique de l'est, Tomo XXIV, $\mathrm{N}^{\circ}$ 1. pp. 47-64.

DUTTON, E.G. (1994): «Atmospherical solar transmission at Mauna Loa». pp. 479-482. In T.A. BODEN, D.P. KAISER, R.J. SEPANSKI and F.W. STOSS (eds.) «Trends'93: A compendium of data on global change» ORNL/CDIAC-65. Carbon Dioxide Information Analysis Center, Oak Ridge National Laboratory, Oak Ridge, Tennessee, U.S.A.

FLOHN, H. (1979): «Notre avenir climatique: un Ocean Artique libre de glaces?». La Météorologie, VI, 16, pp. 35-51.

HAMMANI, S. et alters (1982): «Premires résultats d'une analyse d'altitude de la surface $500 \mathrm{mb}$ de l'Atlantique Nord». La Météorologie, VI, 29-30.

JANSA GUARDIOLA, J.Mª . (1951): «Previsión del tiempo en el Mediterráneo Occidental». Revista de Geofísica, 39.

JONES, P.D. et alters (1985): A grid point surface air temperature data set for the Northern Hemisphere. TR022, DOE/EV/10098. U.S. DOE.

JONES, P.D., WIGLEY, T.M.L. and BRIFFA, K.R. (1994): «Global and hemispheric temperature anomalies-land and marine instrumental records». pp. 603-608. In T.A. BODEN, D.P. KAISER, 
R.J. SEPANSKI and F.W. STOSS (eds.) «Trends '93: A compendium of data on global change» ORNL/CDIAC-65. Carbon Dioxide Information Analysis Center, Oak Ridge National Laboratory, Oak Ridge, Tennessee, U.S.A.

KOLOMEV, M.P. et alt. (1984): «Modelling the Impact of the Eruption of the Volcano el Chichón on climate». Meteorologiya i Hidrologiya, 7.

LAMB, H.H. (1972): «Climate, Past, Present and Future». Metheun, London, 613 pp.

LEGRAND, J.P. (1978): «Fluctuations météorologiques vendages et activié solaire». La Météorologie, 12.

MARTIN, S. et FEYT, G. (1984): «Étude des variations pluviométriques par un modèle d'analyse de la variance a deux facteurs». Revue Géographique de l'est, Tomo XXIV, No 1. pp. 27-46.

MASS, C. and SCHNEIDER, S.H. (1977): «Influence of Sunspot and Volcanic Dust on Longterm Temperature Records Inferred by Statistical Investigations». Journal of Atmospheric Sciences, 34, pp. 1995-2004

MIRCHELL, J.M. et alters (1966): «Climatic Change». W.M.O. Ginebra, 1966.

MORENO, M.C. y MARTÍN VIDE, J. (1986): «Estudio preliminar sobre las tendencias de la precipitación anual en el sur de la Península Ibérica: el caso de Gibraltar». II Simposio sobre el Agua en Andalucía, vol. 1, pp. 37-44, Dep. de Hidrogeología, Universidad de Granada.

NAYA, A. (1989): Problemas de Meteorología superior. I.N.M., Publicación N² 24. 258 pp.

O.M.M. (1990): Guía de prácticas meteorológicas. OMM-Nº 100.

QUEREDA, J. (1986): «Un aspect du régime thermique en Méditerranée occidentale», Met-mar, 131, pp. 14-17.

QUEREDA, J. y MONTON, E. (1994): Las lluvias torrenciales en la Comunidad Valenciana: Interacciones atmósfera-mar. Col. Universitaria, Dip. Provincial, $190 \mathrm{pp}$.

QUEREDA, J. (1985): «Ciclogéneèse et convection dans le Levant Espagnol». La Météorologie, VII, 6, pp. 6-10.

QUEREDA, J. (1987): «Efectos de la actividad volcánica en el clima de Castellón». BSCC, LXIII, pp. 251-260.

QUEREDA, J. (1992): «Significant warming trend in the Spanish Mediterranean». Weather, 47, 7, pp. 274-275.

QUEREDA, J. y MONTON, E. (1994): «¿Hacia un cambio climático?» Universidad Jaume I, 27 pp.

SCHNEIDER, S. H. (1983): «Volcanic Dust Veils and Climate: How Clear is the Connection?». Climatic Change, 5, 2.

SMITH, T. M. (1992): «Sensitivity of terrestrial carbon storage to $\mathrm{CO}_{2}$ induced climate changes: comparison of four scenarios based on G.C.M.». Climatic Change, 21, 4. pp. 367-384.

TANNEHILL, I. (1955): «Is Weather subject to Cycles?». Yearbook of agriculture.

UNESCO (1973): Actes du Colloque de Rome.

UNIPCC (1995): Second Scientific Assessment of Climate.

VOSE, R. S. et al. (1992): The global historical climatology network: long-term monthly temperature, precipitation, sea level pressure and station pressure data. ORNL/CDIAC-53, NDP-041. CDIAC, Oak Ridge, National Laboratory, Oak Ridge, Tennessee, 315 pp.

W.M.O. (1966). «Climatic Change». Technical note, 79, Ginebra. 
